\title{
Discussion on Teaching of Element Chemistry in Inorganic Chemistry
}

\author{
Hu Tianjiao*, Li Gongyi, Wang Qinghua \\ College of Science, National University of Defense Technology, Changsha, China
}

\section{Email address:}

tjhu@nudt.edu.cn (Hu Tianjiao),nudtlgy@163.com (Li Gongyi), qh1002@sina.com (Wang Qinghua)

${ }^{*}$ Corresponding author

\section{To cite this article:}

Hu Tianjiao, Li Gongyi, Wang Qinghua. Discussion on Teaching of Element Chemistry in Inorganic Chemistry. Science Discover. Vol. 5, No. 6, 2017, pp. 476-478. doi: 10.11648/j.sd.20170506.24

Received: September 1, 2017; Accepted: November 15, 2017; Published: December 6, 2017

\begin{abstract}
Base on the characteristics and teaching status of element chemistry, this paper discusses the relationship between teaching strategy and teaching results. In order to motivate the students' interest, cultivate students' creative thinking and improve the quality of teaching in element chemistry curriculum, we combined the teaching idea with the theory content, selected the content of lectures, and sorted out the key points of knowledges.
\end{abstract}

Keywords: Elemental Chemistry, Inorganic Chemistry, Teaching Method

\section{浅谈无机化学中元素化学的教学}

胡天娇”，李公义，王清华

理学院, 国防科技大学, 长沙, 中国

\section{邮箱}

tjhu@nudt.edu.cn（胡天娇）, nudtlgy@163.com(李公义), qh1002@sina.com（王清华）

摘要: 针对无机化学课程中元素化学部分内容庞杂、知识点多、叙述性强等特点, 结合实际教学中的案例, 探讨教学 内容、教学方法和教学手段对提高学生兴趣、培养学生创造性思维以及提高教学质量的影响, 提出与理论内容相结合 的教学理念, 精选授课内容, 梳理知识要点, 以期获得更好的教学效果。

关键词: 元素化学, 无机化学, 教学方法

\section{1. 引言}

无机化学是化学、材料学等专业的学科基础课程。元 素化学是无机化学的重要组成部分, 也是学生学习后续专 业课程的必要基础, 更是完成无机化学课程的核心和重要 环节。但是, 由于元素化学内容庞杂、繁琐、知识点多、 零散、叙述性强, 类似“中药铺”, 使得元素化学长期以来 都是无机化学课程教学的难点。在教学实践中, 教师往往 花费了大量的精力, 却没能收到良好的教学效果。学生也
普遍反映“打开书就能看懂, 合上书又会忘记”。因此, 如 何在较短的学时内让学生掌握数十甚至上百种化学元素 的结构性质变化规律, 是需要深入探索和研究的一项重要 课题[1-4]。

2. 对学生创造性思维能力的培养贯穿教学始终 科技竞争的核心是人才竞争。当今教改研究的主要课 题之一, 就是如何培养创造型、开拓型人才。而落实到具 
体教学活动中, 就是如何培养学生的创造性思维能力, 也 就是在学习与探究活动中发现新事物、提出新见解或解决 新问题的思维能力[5]。

目前中国国内大多数元素化学教材的内容都是按元 素周期表进行编排, 每种元素的单质和化合物总是按存在、 性质、制备、用途的顺序进行讨论，叙述性内容占有大量 篇幅。若在课堂教学中仍简单地照本宣科, 必然无法提高 学生创新思维能力[6]。因此, 在元素化学教学中, 必须重 组、活化传统内容, 在为学生打好基础知识与基本能力的 基础上, 提高他们思考问题、解决问题的能力。例如, 在 元素化学绪论课的教学中, 可通过比较中国古代文明的辉 煌成就和目前在自然科学领域与西方国家的差距, 引导学 生思考中国科技发展在近两个世纪变缓的根本原因。从一 开始就给这门课程定下基调: 元素化学学习不只是孤立的 一个个元素, 在了解具体元素性质的同时, 深入研究和探 讨元素性质变化的根本原因, 并对未知元素或元素的未知 性质进行预测才是本门课程的目的。

敢于质疑是思维创新的前提。在元素化学发展历程中 不乏因传统理论的禁锢而阻碍学科进步的例子。例如“稀 有气体元素是否能形成化合物? ”这一问题就曾困扰了科 学家们数十年。根据传统结构理论, 稀有气体的稳定价电 子构型决定了它不太容易形成化合物。虽然曾有不少知名 科学家根据实验或计算得出稀有气体有可能形成化合物 的推论, 但都因畏惧挑战权威而与这一重大发现失之交臂。 直到1962年, 年仅26岁的英国化学家N. Bartlett受到其他化 学反应的启发, 在相对并不复杂的条件下就获得了第一种 稀有气体化合物, 并由此打开了稀有气体化合物研究的新 篇章。在课程讲授过程中, 可通过类似历史的讲述向学生 展示突破固有观念在科学研究中的重要性, 帮助学生逐步 建立起敢于质疑、善于提问的学习态度。

此外, 教师在整个的教学过程中应着力于为学生提供 积极思维的机会, 创造刺激学生积极思维的情境。无机化 学作为一门发展最早、研究最深入的学科, 很多理论都已 被广泛接受。但随着物质结构分析测试技术的发展, 一些 传统理论受到了新的挑战。比如 $\mathrm{NO}_{2}$ 的结构, 过去一直认 为它存在 4 个电子的离域大 $\pi$ 键, 近几年的研究发现它只存 在 3 个电子的离域大 $\pi$ 键。再如, 化学界对同一主族的最高 价含氧酸氧化性为锯齿状变化, 对物质呈现颜色的原因等 现象一直有不同的解释，并无统一的理论。教师可在讲授 过程中可将这些具有争议的问题提出, 引导学生利用所学 知识对不同观点进行分析。也可以将类似问题留为作业, 让学生课后查阅资料提出自己的想法。这样不仅加深了学 生对元素知识的理解, 也锻炼了学生分析问题、解决问题 的能力。

\section{3. 将基础理论融入到元素化学教学中}

随着物质结构研究手段和相应理论的发展, 对化学物 质的研究, 逐渐由“见著而知微”的传统认知方式, 转而成 为兼有 “见著而知微”和“见微而知著”相结合的方式。其显 著的标志之一, 就是物质结构和化学热力学等理论在元素 化学中的应用[7]。但传统教学方式通常将理论与元素知识
分开讲述。这样往往会使学生在学习理论部分时觉得枯燥 无聊、不知有何用处, 在学习元素部分时又觉得杂乱无章、 无规律可循。所以, 在无机化学的教学中应该将这两部分 内容有机结合起来, 以基础理论为框架, 将每一个元素的 结构、性质、用途套入这个框架中进行学习。这样不仅可 以丰富理论部分的内容, 加深学生对基础理论的理解, 同 时也可以梳理元素部分的知识, 使元素化学的学习有章可 循。

例如电极电势和电离能都是衡量元素稳定性的重要 参数, 但两者讨论的化学过程是完全不同的。电极电势是 衡量金属原子失去电子形成水合离子的倾向, 其大小与电 离能、升华热和水合能有关。而电离能的大小是孤立原子 电子结构稳定性的定量量度, 只能衡量气态基态原子失去 电子变成气态基态阳离子的难易程度 [8]。若将两者混为一 谈, 很可能得出相互矛盾的结论。在元素化学的教学过程 中, 可把此问题与锂元素的学习结合起来。通过锂的标准 电极电势出现反常这一现象, 引导学生对上述两个参数进 行比较, 讨论电离能、升华热、水合能对能量过程的贡献 程度, 并与结构因素相关联, 深入了解其变化规律。

\section{4. 精选教学内容, 注重讲授方式}

元素无机化学的内容庞杂, 涉及面广, 元素周期表中 的一百多种元素的单质及其化合物的各种性质均有大量 的化学反应来体现。如果所有内容全部在课堂上讲授, 一 方面, 由于面面俱到, 势必不深不透, 学生难以记忆和掌 握; 另一方面, 元素部分内容往往是叙述性材料, 如果照 本宣科, 则教起来平淡无奇, 学起来千篇一律、枯燥无味, 会影响教学效果。因此, 在教学过程中应精心选取讲授内 容, 并探索新的教学方法, 提高学生兴趣, 强化教学效果。

\section{1. 教学内容的精炼}

首先, 教学内容应该传统性与先进性并重。对于那些 在化学工业上占有举足轻重地位的传统产品, 如常见化合 物中的重要化工原料及产品, 它们的来源、性质、制备方 法、用途等应有所介绍[8]。但随着科学技术的不断发展, 很多传统工艺已经被新型的技术所取代。例如传统光学相 机的洗相技术在早期教材中是非常重要的教学案例, 其定 影、显影过程都涉及非常典型的化学反应。但随着数码相 机的普及, 传统光学相机的使用率已经很低, 这部分内容 很难再引起 90 甚至 00 后学生的共鸣。故在现在的教学中, 可以适当减少这些内容的讲授。另一方面, 随着物质制备 技术的不断发展, 又有很多全新的重要物质涌现出来。比 如自2004年石墨烯被成功制备出来后, 三维的金刚石和石 墨、“二维”的石墨烯、一维的碳纳米管、零维的富勒球组 成了完整的碳系家族。有关碳元素的化学引起了世界各国 研究人员的极大兴趣, 增加这些内容的学习可以开拓学生 眼界, 激发他们的学习动机。因此, 元素化学的教学应紧 扣时代脉搏, 及时调整教学内容, 并引导学生学会自我发 展[9]。

其次, 教学内容的选择应考虑学生的专业特点, 因材 施教。无机化学不仅是化学各专业的基础必修课, 同样也 
是材料学、医学、生物学等专业的重要基础课程。针对不 同专业的学生, 课程教学的侧重点应有不同。如在对材料 学专业学生的授课过程中, 应着重于介绍物质材料性能与 其结构之间的关系, 并根据本校材料学科领域的科研现状, 在学习具体元素时引入相关材料学科的知识, 为学生后续 课程学习和将来的毕业设计打下基础。对于医学、生物学 专业的学生, 可在教学中穿插介绍一些与元素生理效应有 关的著名事件和人物, 以及人体必需元素含量异常所导致 的疾病, 将元素的致病机理导入其中, 并有意识地渗透化 学生物学、分子生物学、神经生理学等相关交叉学科的知 识, 扩大学生的知识面。

此外, 还可在课程教学中适当引入热点新闻和趣味化 学史, 在提高学生兴趣的同时, 将正确的人生观和价值观 潜移默化地传递给学生。校园投毒案是近期非常热门的一 个话题, 结合此话题可引入化学元素与人体健康的专题讨 论。指导学生通过课外读物、科技文献的查询, 系统探讨 化学元素在生命体中的平衡协调作用。通过讲述合成氨工 业的建立者Fritz Haber、四乙铅的发明人Midgley等人的生 平事迹, 教育学生“科学是一柄双刃剑”, 要用自己的技能 为社会发展做出积极的贡献, 不能只顾眼前利益, 成为历 史的罪人。

\section{2. 授课方式的改进}

多媒体技术能够将文字、声音、图形和图像融为一体, 是激发学生学习兴趣的重要手段。在元素化学的学习中, 多媒体资源的合理利用更为重要。因为在实际生活中, 学 生能够直接接触到的单质、化合物数量有限, 仅通过口头 或文字描述很难建立起直观的印象。此时采用图片、视频 等方式进行教学往往能达到事半功倍的效果。但由于多媒 体教学自身的特点, 容易给学生带来过重负担, 造成视觉 的疲劳和思维束缚[10]。因此, 在多媒体教学过程中, 一 方面应注意加强对图片、视频进行剖析总结, 另一方面还 应结合板书对重点、难点问题进行教学。这样, 既可以控 制课程进度, 又可增加与学生交流的机会, 弥补了单纯使 用多媒体教学的不足。

元素周期表中的元素有一百多种, 需要重点学习的元 素也有数十种, 要将这些元素的性质一一列举是不现实的, 但结构相似的物质往往具有相似的性质, 所以在授课过程 中可以引导学生对物质的某些性质进行归纳总结, 并对关 键性质设置专题进行重点讲授。例如对于非金属元素来说, 其化合物主要包括三类: 氢化物、含氧酸以及含氧酸盐。 其中, 氢化物的水溶液和含氧酸最重要的性质就是其酸性, 设置专题对此进行系统讨论有助于加深学生对非金属原 子结构的理解和对元素化合物性质的判断能力。金属元素 的提炼和制备是化学化工领域非常重要的基础知识, 但由 于发展历史久远, 内容庞杂、信息量大。该部分内容进行
归纳总结可帮助学生梳理思路, 为将来的学习和工作打下 坚实基础。

\section{5. 结论}

元素化学本是一门充满趣味的科学, 但过于丰富的内 容却给学生造成过重负担, 使之无法体会课程学习的乐趣。 因此, 在元素化学的教学中必须重视技巧和方法, 使学生 能够在愉悦中获取知识。只有这样, 才能展示出化学学科 的独特鬼力。

\section{致谢}

本文为国防科技大学校级教改课题《全程导师引导式 融合实战需求的团队教学模式研究》(U2015002)的阶段性 成果之一。

\section{参考文献}

[1] 周小霞, 肖湘, 季卫刚, 赵华文, 赵先英. 无机化学元素部 分教学体会 $[J]$. 大学教育, 2016,(01):102-103。

[2] 陈三平, 谢钢, 杨奇, 王尧宇, 高胜利. 加强元素化学教学 的改革和实践 $[J]$. 高等理科教育, 2012,3:119-122。

[3] 吕仁江, 于海霞, 马玲, 白丽明, 陈世界. 探索提高无机元 素化学课堂教学质量的途径和方法 [J]. 化工时刊, 2015,29(11):51-53。

[4] 庄晓娟, 韩明梅, 探讨PBL教学法在无机化学元素部分教 学中的应用 [J]. 大学化学, 2016,31(12):13-16。

[5] 张欢, 王伟, 杨定明, 戴亚堂. 从大学生能力培养视角谈无 机元素化学教学 [J]. 广州化工, 2015,43(21):203-205。

[6] 马占营, 徐维霞, 耿薇, 高奕红. 无机化学中元素化学教学 改革的探讨 $[J]$. 教育视点, 2010,4:9-10。

[7] 秦雪儿. 关于“元素周期表”中学化学史教学的几点思考 [J]. 亚太教育, 2016,(10):63。

[8] 刘新锦, 朱亚先, 高飞编著. 无机元素化学 [M]. 北京: 科学 出版社, 2010。

[9] 严新. 无机及分析化学中的元素化学教学探讨 [J]. 山东化工, 2011,40:86-88。

[10] 蒋育澄, 李淑妮, 翟全国. 元素的生物效应在元素化学教学 中的渗透与融合, 2009,24(4):35-40。 